\title{
RADON-FOURIER TRANSFORMS ON SYMMETRIC SPACES AND RELATED GROUP REPRESENTATIONS ${ }^{1}$
}

BY S. HELGASON

Communicated by G. D. Mostow, May 7, 1965

In $\$ 2$ we announce some results in continuation of [10], connected with the Radon transform. $\$ 1$ deals with tools which also apply to more general questions and $\S \S 2-3$ contain some applications to group representations. A more detailed exposition of $\$ 2$ appears in Proceedings of the U. S.-Japan Seminar in Differential Geometry, Kyoto, June, 1965.

1. Radial components of differential operators. Let $V$ be a manifold, $v$ a point in $V$ and $V$, the tangent space to $V$ at $v$. Let $G$ be a Lie transformation group of $V$. A $C^{\infty}$ function $f$ on an open subset of $V$ is called locally invariant if $X f=0$ for each vector field $X$ on $V$ induced by the action of $G$.

Suppose now $W$ is a submanifold of $V$ satisfying the following transversality condition:

$$
\text { For each } w \in W, V_{w}=W_{w}+(G \cdot w)_{w} \quad \text { (direct sum). }
$$

If $f$ is a function on a subset of $V$ its restriction to $W$ will be denoted $\bar{f}$.

Lemma 1.1. Let $D$ be a differential operator on $V$. Then there exists a unique differential operator $\Delta(D)$ on $W$ such that

$$
(D f)^{-}=\Delta(D) \bar{f}
$$

for each locally invariant $f$.

The operator $\Delta(D)$ is called the radial component of $D$. Many special cases have been considered (see e.g. $[1, \S 2],[4, \S 5],[5, \S 3],[7, \S 7]$, [8, Chapter IV, \$§3-5]).

Suppose now $d v$ (resp. $d w$ ) is a positive measure on $V$ (resp. $W$ ) which on any coordinate neighborhood is a nonzero multiple of the Lebesgue measure. Assume $d g$ is a bi-invariant Haar measure on $G$. Given $u \in C_{c}^{\infty}(G \times W)$ there exists [7, Theorem 1] a unique $f_{u} \in C_{c}^{\infty}(G \cdot W)$ such that

$$
\int_{G \times W} F(g \cdot w) u(g, w) d g d w=\int_{\nabla} F(v) f_{u}(v) d v \quad\left(F \in C_{c}^{\infty}(G \cdot W)\right) .
$$

Let $\phi_{u} \in C_{c}^{\infty}(W)$ denote the function $w \rightarrow \int u(g, w) d g$.

${ }^{1}$ Work supported in part by the National Science Foundation, NSF GP-2600. 
Theorem 1.2. Suppose $G$ leaves dv invariant. Let $T$ be a G-invariant distribution on $G \cdot W$. Then there exists a unique distribution $\bar{T}$ on $W$ such that

$$
\bar{T}\left(\phi_{u}\right)=T\left(f_{u}\right), \quad u \in C_{c}^{\infty}(G \times W) .
$$

If $D$ is a G-invariant differential operator on $V$ then

$$
(D T)^{-}=\Delta(D) \bar{T} \text {. }
$$

The proof is partly suggested by the special case considered in [7, §9]. See also $[12, \S 4]$.

2. The Radon transform and conical distributions. Let $G$ be a connected semisimple Lie group, assumed imbedded in its simply connected complexification. Let $K$ be a maximal compact subgroup of $G$ and $X$ the symmetric space $G / K$. Let $G=K A N$ be an Iwasawa decomposition of $G$ ( $A$ abelian, $N$ nilpotent) and let $M$ and $M^{\prime}$, respectively, denote the centralizer and normalizer of $A$ in $K$. The space $\Xi$ of all horocycles $\xi$ in $X$ can be identified with $G / M N[10, \S 3]$. Let $\boldsymbol{D}(X)$ and $\boldsymbol{D}(\boldsymbol{\Xi})$ denote the algebras of $G$-invariant differential operators on $X$ and $\Xi$, respectively; let $S(A)$ denote the symmetric algebra over the vector space $A$ and $I(A)$ the set of elements in $S(A)$ which are invariant under the Weyl group $W=M^{\prime} / M$. There are isomorphisms $\Gamma$ of $D(X)$ onto $I(A)[6$, p. 260], [9, p. 432] and $\hat{\Gamma}$ of $D(\Xi)$ onto $S(A)[10$, p. 676].

The Radon transform $f \rightarrow \vec{f}\left(f \in C_{c}^{\infty}(X)\right)$ and its dual $\phi \rightarrow \check{\phi}\left(\phi \in C^{\infty}(\Xi)\right)$ are defined by

$$
\ddot{f}(\xi)=\int_{\xi} f(x) d m(x), \quad \check{\phi}(x)=\int_{\check{x}} \phi(\xi) d \mu(\xi) \quad(x \in X, \xi \in \Xi)
$$

where $d m$ is the measure on $\xi$ induced by the canonical Riemannian structure of $X, \check{x}$ is the set of horocycles passing through $x$ and $d \mu$ is the measure on $\check{x}$ invariant under the isotropy subgroup of $G$ at $x$, satisfying $\mu(\check{x})=1$. The easily proved relation

$$
\int_{X} f(x) \check{\phi}(x) d x=\int_{\Xi} f(\xi) \phi(\xi) d \xi \quad\left(f \in C_{c}^{\infty}(X), \phi \in C_{c}^{\infty}(\Xi)\right)
$$

$d x$ and $d \xi$ being $G$-invariant measures on $X$ and $\Xi$, respectively, suggests immediately how to extend the integral transforms above to distributions.

Let $\mathbb{B}$ and $\mathfrak{A}$ be the Lie algebras of $G$ and $A$, respectively, and $\mathfrak{A}^{*}$ 
the dual space of $\mathfrak{A}$. Let $\lambda \rightarrow c(\lambda)$ be the function on $\mathfrak{A}^{*}$ giving the Plancherel measure $|c(\lambda)|^{-2} d \lambda$ for the $K$-invariant functions on $X$ (Harish-Chandra [6, p. 612]). Let $j$ be the operator on rapidly decreasing functions on $A$ which under the Fourier transform on $A$ corresponds to multiplication by $c^{-1}$. Let $\rho$ denote the sum (with multiplicity) of the restricted roots on $\mathfrak{A}$ which are positive in the ordering given by $N$. Let $e^{\rho}$ denote the function on $\Xi$ defined by $e^{\rho}(k a M N)=\exp [\rho(\log a)](k \in K, a \in A)$. Viewing $\Xi$ as a fibre bundle with base $K / M$, fibre $A[10$, p. 675] we define the operator $\Lambda$ on suitable functions $\phi$ on $\Xi$ by $\left(e^{\rho} \Lambda \phi\right) \mid F=j\left(\left(e^{\rho} \phi\right) \mid F\right)$, where $\mid F$ denotes restriction to any fibre $F$. Similarly, the complex conjugate of $c^{-1}$ determines an operator $\bar{\Lambda}$. By means of the Plancherel formula mentioned one proves (cf. $[11, \S 6])$.

THEOREM 2.1. There exist constants $c, c^{\prime}>0$ such that

$$
\begin{gathered}
\int_{X}|f(x)|^{2} d x=c^{\prime} \int_{\Xi}|\Lambda \hat{f}(\xi)|^{2} d \xi \\
f=c(\Lambda \bar{\Lambda} \hat{f})^{-}
\end{gathered}
$$

for all $f \in C_{c}^{\infty}(X)$.

If all Cartan subgroups of $G$ are conjugate, the operators $j$ and $\Lambda$ are differential operators ( $c^{-1}$ is a polynomial). Considering $j j$ is an element in $I(A)$ we put $\square=\Gamma^{-1}(j \bar{j}) \in D(X)$. Then (3) can be written in the form

$$
f=c \square\left((\check{f})^{\vee}\right), \quad f \in C_{c}^{\infty}(X),
$$

which is more convenient for applications [10, \$7]. For the case when $G$ is complex a formula closely related to (3) was given by GelfandGraev $[2, \S 5.5]$.

Let $x_{0}$ and $\xi_{0}$ denote the origins in $X$ and $\Xi$, respectively. The space $B=K / M$ can be viewed as the set of Weyl chambers emanating from $x_{0}$ in $X$. If $\xi=k a \cdot \xi_{0}(k \in K, a \in A)$ we say that the Weyl chamber $k M$ is normal to $\xi$ and that $a$ is the complex distance from $x_{0}$ to $\xi$. If $x \in X$, $b \in B$ let $\xi(x, b)$ be the horocycle with normal $b$ passing through $x$, and let $A(x, b)$ denote the complex distance from $x_{0}$ to $\xi(x, b)$.

ThEOREM 2.2. For $f \in C_{c}^{\infty}(X)$ define the Fourier transform $\tilde{f}$ by

$$
\tilde{f}(\lambda, b)=\int_{X} f(x) \exp [(-i \lambda+\rho)(A(x, b))] d x \quad\left(\lambda \in \mathfrak{Y}^{*}, b \in B\right) .
$$

\section{Then}




$$
\begin{aligned}
f(x) & \left.=\int_{\mathscr{x * X B}} \tilde{f}(\lambda, b) \exp (i \lambda+\rho)(A(x, b))\right]|c(\lambda)|^{-2} d \lambda d b \\
\int_{X}|f(x)|^{2} d x & =\int_{\mathscr{Q} * \times B}|\tilde{f}(\lambda, b)|^{2}|c(\lambda)|^{-2} d \lambda d b
\end{aligned}
$$

where $d b$ is a suitably normalized $K$-invariant measure on $B$.

REMARKS. (i) In view of the analogy between horocycles in $X$ and hyperplanes in $R^{n}$ formula (4) corresponds exactly to the Fourier inversion formula in $R^{n}$ when written in polar coordinate form.

(ii) If $f$ is a $K$-invariant function on $X$, Theorem 2.2 reduces to Harish-Chandra's Plancherel formula [6, p. 612]. Nevertheless, Theorem 2.2 can be derived from Harish-Chandra's formula.

(iii) A "plane wave" on $X$ is by definition a function on $X$ which is constant on each member of a family of parallel horocycles. Writing (4) in the form

$$
f(x)=\int_{B} f_{b}(x) d b
$$

we get a continuous decomposition of $f$ into plane waves. On the other hand, if we write (4) in the form

$$
f(x)=\int_{\mathfrak{I}^{*}} f_{\lambda}(x)|c(\lambda)|^{-2} d \lambda
$$

we obtain a decomposition of $f$ into simultaneous eigenfunctions of all $D \in D(X)$.

We now define for $\Xi$ the analogs of the spherical functions on $X$.

Definition. A distribution (resp. $C^{\infty}$ function) on $\Xi=G / M N$ is called conical if it is (1) $M N$-invariant; (2) eigendistribution (resp. eigenfunction) of each $D \in D(\Xi)$.

Let $\xi_{0}=M N, \xi^{*}=m^{*} M N$, where $m^{*}$ is any element in $M^{\prime}$ such that the automorphism $a \rightarrow m^{*} a m^{*-1}$ of $A$ maps $\rho$ into $-\rho$. By the Bruhat lemma, $\boldsymbol{Z}$ will consist of finitely many $M N A$-orbits; exactly one, namely $\Xi^{*}=M N A \cdot \xi^{*}$, has maximum dimension and given $\xi \in \Xi^{*}$ there exists a unique element $a(\xi) \in A$ such that $\xi \in M N a(\xi) \cdot \xi^{*}$ $[10$, p. 673]. Using Theorem 1.2 we find:

TheOREM 2.3. Let $T$ be a conical distribution on $\Xi$. Then there exists $a \psi \in C^{\infty}\left(\Xi^{*}\right)$ such that $T=\psi$ on $\Xi^{*}$ and a linear function $\mu: \mathfrak{A} \rightarrow C$ such that

$$
\psi(\xi)=\psi\left(\xi^{*}\right) \exp [\mu(\log a(\xi))]
$$

$\left(\xi ; \in \Xi^{*}\right)$. 
In general $\psi$ is singular on the lower-dimensional $M N A$-orbits. However, we have:

TheOREM 2.4. Let $\mu: \mathfrak{A} \rightarrow \boldsymbol{C}$ be a linear function and let $\psi \in C^{\infty}\left(\Xi^{*}\right)$ be defined by (5). Then $\psi$ is locally integrable on $\Xi$ if and only if

$$
\operatorname{Re}(\langle\alpha, \mu+\rho\rangle)>0 \quad(\operatorname{Re}=\text { real part })
$$

for each restricted root $\alpha>0$; here $\langle$,$\rangle denotes the inner product on \mathfrak{A}^{*}$ induced by the Killing form of (5. If (6) is satisfied then $\psi$, as a distribution on $\Xi$, is a conical distribution.

THEOREM 2.5. The conical functions on $\Xi$ are precisely the functions $\psi$ given by (5) where for each restricted root $\alpha>0$,

$$
\frac{\langle\mu, \alpha\rangle}{\langle\alpha, \alpha\rangle} \text { is an integer } \geqq 0 .
$$

Definition. A representation $\pi$ of $G$ on a vector space $E$ will be called (1) spherical if there exists a nonzero vector in $E$ fixed by $\pi(K)$; (2) conical if there exists a nonzero vector in $E$ fixed by $\pi(M N)$.

The correspondence between spherical functions on $X$ and spherical representations is well known. In order to describe the analogous situation for $\Xi$, for an arbitrary function $\phi$ on $\Xi$, let $E_{\phi}$ denote the vector space spanned by the $G$-translates of $\phi$ and let $\pi_{\phi}$ denote the natural representation of $G$ on $E_{\phi}$.

TheOREM 2.6. The mapping $\psi \rightarrow \pi_{\psi}$ maps the set of conical functions on $\mathbf{\Xi}$ onto the set of finite-dimensional, irreducible conical representations of $G$. The mapping is one-to-one if we identify proportional conical functions and identify equivalent representations. Also

$$
\psi\left(g \cdot \xi_{0}\right)=\left(\pi_{\psi}\left(g^{-1}\right) e, e^{\prime}\right),
$$

where e and $\mathrm{e}^{\prime}$, respectively, are contained in the highest weight spaces of $\pi_{\psi}$ and of its contragredient representation. Finally, $\mu$ in (5) is the highest weight of $\pi_{\psi}$.

Corollary 2.7. Let $\pi$ be a finite-dimensional irreducible representation of $G$. Then $\pi$ is spherical if and only if it is conical.

The highest weights of these representations are therefore characterized by (7). Compare Sugiura [13], where the highest weights of the spherical representations are determined.

3. The case of a complex $G$. If $G$ is complex, $M$ is a torus and some of the results of $\$ 2$ can be improved. Let $\mathfrak{S}$ be a Cartan subalgebra 
of $\mathbb{3}$ containing $\mathfrak{A}$ and $H$ the corresponding analytic subgroup of $G$. Now we assume $G$ simply connected.

Let $D(G / N)$ denote the algebra of all $G$-invariant differential operators on $G / N$. Let $\nu_{0}, \nu^{*} \in G / N$ be constructed similarly as $\xi_{0}$ and $\xi^{*}$ in $\$ 2$. Then $\$ 1$ applies to the submanifold $W=H \cdot \nu^{*}$ of $V=N H \cdot \nu^{*}$ and for each differential operator $D$ on $G / N, \Delta(D)$ is defined and can be viewed as a differential operator on $H$.

THEOREM 3.1. The mapping $D \rightarrow \Delta(D)$ is an isomorphism of $D(G / N)$ onto the (real) symmetric algebra $S(\mathfrak{S})$. In particular, $D(G / N)$ is commutative.

As a consequence one finds that the $N$-invariant eigenfunctions $f \in C^{\infty}(G / N)$ of all $D \in D(G / N)$ have a representation analogous to (5) in terms of the characters of $H$. Let $E_{f}$ denote the vector space spanned by the $G$-translates of $f$ and let $\pi_{f}$ be the natural representation of $G$ on $E_{f}$.

THEOREM 3.2. The mapping $f \rightarrow \pi_{f}$ is a one-to-one mapping of the set of $N$-invariant holomorphic eigenfunctions of all $D \in D(G / N)$ (proportional $f$ identified) onto the set of all finite-dimensional ${ }^{2}$ irreducible holomorphic representations of $G$ (equivalent representations identified). Moreover

$$
f\left(g \cdot \nu_{0}\right)=\left(\pi_{f}\left(g^{-1}\right) e, e^{\prime}\right)
$$

where e and $\mathrm{e}^{\prime}$, respectively, are contained in the highest weight spaces of $\pi_{f}$ and of its contragredient representation.

\section{REFERENCES}

1. F. A. Berezin, Laplace operators on semisimple Lie groups, Trudy Moskov. Mat. Obšc. 6 (1957), 371-463; Amer. Math. Soc. Transl. 21 (1962), 239-299.

2. I. M. Gelfand and M. I. Graev, The geometry of homogeneous spaces, group representations in homogeneous spaces and questions in integral geometry related to them. I, Trudy Moskov. Mat. Obšc. 8 (1959), 321-390; Amer. Math. Soc. Transl. 37 (1964), 351-429.

3. R. Godement, $A$ theory of spherical functions. I, Trans. Amer. Math. Soc. $\mathbf{7 3}$ (1952), 496-556.

4. Harish-Chandra, The characters of semisimple Lie groups, Trans. Amer. Math. Soc. 83 (1956), 98-163.

5. - Differential operators on a semisimple Lie algebra, Amer. J. Math. 79 (1957), 87-120.

6. - Spherical functions on a semisimple Lie group. I, II, Amer. J. Math. 80 (1958), 241-310, 553-613.

2 Compare the problem indicated in $[3$, p. 553]. 
7. - Invariant distributions on Lie algebras, Amer. J. Math. 86 (1964), 271309.

8. S. Helgason, Differential operators on homogeneous spaces, Acta Math. 102 (1959), 239-299.

9. - Differential geometry and symmetric spaces, Academic Press, New York, 1962.

10. - Duality and Radon transform for symmetric spaces, Amer. J. Math. 85 (1963), 667-692.

11. - A duality in integral geometry; some generalizations of the Radon transform, Bull. Amer. Math. Soc. 70 (1964), 435-446.

12. P-D. Methée, Sur les distributions invariantes dans le groupe des rotations de Lorentz, Comment. Math. Helv. 28 (1954), 225-269.

13. M. Sugiura, Representations of compact groups realized by spherical functions on symmetric spaces, Proc. Japan Acad. 38 (3) (1962), 111-113.

INSTITUTE FOR ADVANCEd StUdy 